\title{
COLLECTIVE EXCITATION SPECTRA OF TRANSITIONAL EVEN NUCLEI
}

\author{
P. Quentin \\ Lawrence Livermore National Laboratory \\ Livermore, California 94550 \\ E \\ C.S.N.S.M., Bat. 105 \\ $\mathcal{E}$ \\ I. Deloncle, J. Libert \\ C.S.N.S.M., Bat. 105 \\ J. Sauvage \\ Division de Recherche Expérimentale \\ Institut de Physique Nucléaire \\ 91405 Osray-Campus, France
}

This paper was prepared for submittal to the

Capture Gamma-Ray Spectroscopy Conference Proceedings

Asilomar, Pacific Grove, California

14-19 October 1990

November 6,1990

This is a preprint of a paper intended for publication in a journal or proceedings. Since changes may be made before publication, this preprint is made available with the understanding that it will not be cited or reproduced without the permission of the author.
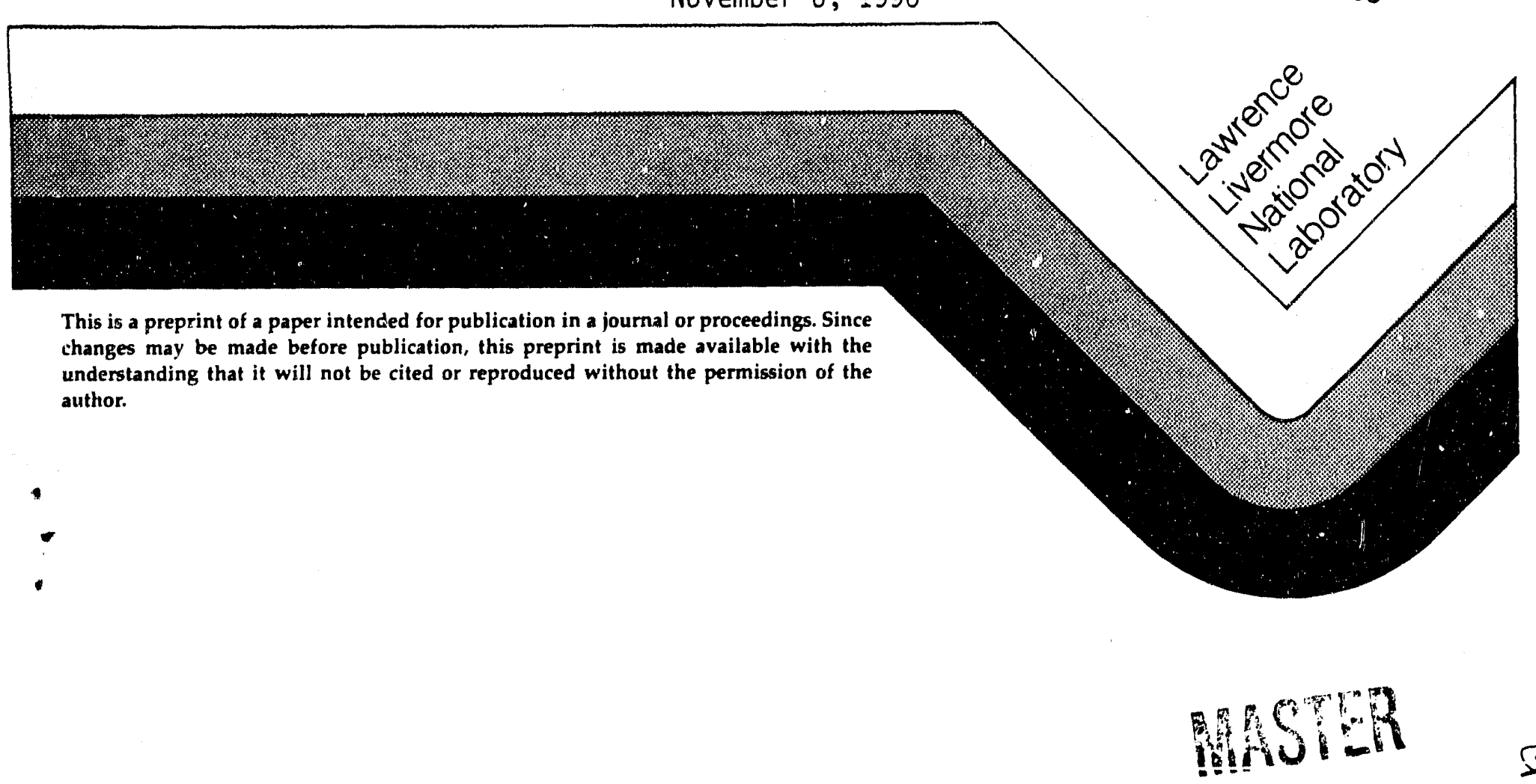
DISCI.AIMER

This document was prepared as an? =irount of work spenswred by an agencs of the United Situtes Government. Neither the U!nited States Government nor the Liniversity of (alifornia nor any of their employees, makes any narrants. express or implied. or assumes any lezal liability or responsibility for the accuracy. completeness, or usefulness of any informution, apparatus, product, or process disclosed, or represents that its use would not infringe privately owned rights. Reference herein in any specific commersial products, process. or service by trade name, trademark, munufacturer. or otherwise, does not necessarily constitute or imply its endorsement. recommendation. on favoring by the United States Government or the University of California. The views and opinions of authors expressed herein do not necessarily state or reflect those of the United States Government or the University of California, and shall not be used for advertising or product endorsement purposes. 


\title{
COLLECTIVE EXCITATION SPECTRA \\ OF TRANSITIONAL EVEN NUCLEI +
}

\author{
P. Quentin \\ Department of Physics. \\ Lawrence Livermore National I,aboratory. \\ Livermure, CA 94550, LSA \\ and \\ C.S.N.S.M. ${ }^{\dagger}$, Bat. 104 , \\ 91405 Orsay-Campus, France \\ I. Deloncle, J. Libert \\ C.S.N.S.M., Bat. 104 \\ 9140.5 Orsay-Campus, France \\ J. Sauvage \\ 91405 Orsay-Campus, France
}

Division de Recherche Expérimentale $\ddagger$ Institut de Physique Nucléaire,

\section{INTRODUCTION}

This talk is dealing with the nuclear low energy collective motion as described in the context of microscopic versions of the Bohr Hamiltonian ${ }^{1)}$. Two different ways of building microscopically Bohr collective Hamiltonians will be sketched in Section II: one within the framework of the Generator Coordinate Method, the other using the Adiabatic Time-Dependent Hartree-FockBogolyubov approximation. A sample of recent results will be presented in Section III which pertains to the description of transitional even nuclei and to the newly revisited phenomenon of superdeformation at low spin.

We will only consider here the five quadrupole degrees of freedom which are (after a well-known transformation from the lab frame to a body fixed incrtial frame) three Euler angles and the usual $\beta$ and $;$ parameters ${ }^{11}$.

t Laboratoire propre du CXRS

‡ Laboratoire associe au CXRS 
The classical Bohr Hamiltonian $H$ is the sum of a potential energy $V$ and of a kinetic energy $T$ splitted into two parts, a vibrational energy $T_{v}$ and a rotational en a $T_{r}$ defined with obrious notation as:

$$
T_{q}=\frac{1}{2} \sum_{i=1}^{2} B_{i j} \dot{q}_{i} \dot{q}_{j}
$$

( with $q_{1}, q_{2}$ standing for $\beta, \gamma$ ) and

$$
T_{p}=\frac{1}{2} \sum_{i=1}^{3} y_{i} \omega_{i}^{2}
$$

In order to get a quantal doscription of the nuclear collective modes, one should quantize $\mathrm{H}$, a task for which there are no well-defined prescriptions in the most general case. Using for instance the Pauli prescription, cne would get, for the vibrational kinetic energy operator acting on a collective wavefunction:

$$
T_{r}=-\frac{\hbar^{2}}{2} \sum_{i, j=1}^{2}(\operatorname{det} B)^{-1 / 2} \frac{\partial}{\partial q_{i}}(\operatorname{det} B)^{1 / 2}\left(B^{-1}\right)_{i j} \frac{\partial}{\partial q_{j}} \text { (3) }
$$

whereas the rotational kinetic energy would be simply given as

$$
T_{r}=\frac{1}{2} \sum_{i=1}^{2} \hat{I}_{i}^{2} / \gamma_{i}
$$

When actually dealing with the Bohr Hamiltonian, one has to take proper care of the symmetries inherent to the problem ${ }^{1,2)}$ and then one merely considers one sextant in the $(\beta, \gamma)$ plane.

The diagonalization of the most general Bohr Hamiltonian is currently performed either by a finite difference treatment of the derivatives which are associated to $T_{r}{ }^{2,3)}$ or by projection onto a suitably chosen (and symmetrized) basist).

Apart from the quantization problem, the main theoretical task will consist in evaluating microscopically the ingredients of the classical/quantal Bohr Hamiltonian. There are indeed, seven scalar functions of $\beta$ and $\gamma$ which need to be determined: the potential $\mathrm{V}$, three moments of inertia $\left(\Im_{1}, \Im_{2}, \Im_{3}\right)$ and three mass parameters $\left(B_{\beta, \beta}, B_{\beta, \gamma}, B_{\gamma, \gamma}\right)$.

\section{MICROSCOPIC DERIVATIONS OF BOHR COLLECTIVE HAMILTONIANS}

\section{I DERIVATION FROM THE GENER ATOR COORDINATE METHOD}

The Generator Coordinate Method ( GCM ) starts from a set of Slater determinants or BCS wavefunctions $\mid \Phi_{q}>$ depending on a coordinate $q$ ( in What follows we will restrict for the sake of clarity, to the simple case of a single 
such coordinate). 'The trial wavefunction $\left|\Psi_{\alpha}\right\rangle$ to be used in a variational approach is built as a linear combination of such wavefunctions

$$
\left|\Psi_{\alpha}\right\rangle=\int d q f_{\alpha}(q)\left|\Phi_{q}\right\rangle
$$

(5).

The mixing amplitudes $f_{a}(q)$ are determined th ought the variational printciple by the following Hill-Wheeler ${ }^{5)}$ equation

$$
\int d q^{\prime}<\Phi_{q}\left|H-E_{\alpha}\right| \Phi_{q^{\prime}}>f_{\alpha}\left(q^{\prime}\right)=0
$$

(6)

or in a condensed vector form as

$$
\mathcal{H} g_{\alpha}=E_{\alpha} \sqrt{ } g_{\alpha}
$$

(7)

where

$$
\begin{aligned}
& \mathcal{H}\left(q, q^{\prime}\right)=\left\langle\Phi_{q}|H| \Phi_{q^{\prime}}\right\rangle \\
& \mathcal{N}\left(q, q^{\prime}\right)=\left\langle\Phi_{q} \mid \Phi_{q^{\prime}}\right\rangle
\end{aligned}
$$

(8).

Whereas $f_{\alpha}$ represents in some way the amount of correlations associated with the degree of freedom which the set $\left(\left|\Phi_{q}\right\rangle\right)$ is supposed to describe, its interpretation as a collective wavefunction suffers from the fact that els. (6-7) are not standard eigenvalue equations due to the use of non-orthogonal $\left|\Phi_{q}\right\rangle$ states. One therefore currently switches to $2 n$ alternate representation $\left.{ }^{6}\right)$. For that purpose, one may notice that the matrix $\mathcal{N}$ being real (if the $\left|\Phi_{q}\right\rangle$ states are even with respect to time-reversal) and symmetrical, it can be diagonalized. As a norm matrix moreover, it has nonnegative eigenvalues. One first rejects eigenvectors with zero eigenvalues whose existence reflects the non-linearly independent character of the initial set of $\left|\Phi_{q}\right\rangle$ st rates. This allows one therefore, to consider $\mathcal{N}^{-1}$. One also can evaluate $\mathcal{N}^{1 / 2}$ and $\mathcal{N}^{-1 / 2}$ in a straight forward fashion from the so-restricted eigensolutions of $\mathcal{N}$.

The collective wavefunction will then be defined as

$$
g_{\alpha}=N^{-1 / 2} f_{\alpha}
$$

(9)

or more explicitly

$$
g_{\alpha}(r)=\int d q\left(w^{1 / 2}\right)_{q r} f_{\alpha}(q)
$$

(10).

It is then possible to onthonomalize the $g$ a functions and the Hill- Wheeler equation (T) will become

$$
h g_{\alpha}=E_{\alpha} g_{\alpha}
$$

(11)

where the Hamiltonian matrix $h$ is given in terms of the original $\mathrm{H}$ by $h=N^{-1 / 2} H \mathcal{N}^{-1 / 2}$

(12).

Page 3 
Now starting from the modified Hill-Whecler cquation (11) we will sketch the derivation of the underlying Boln hamiltonian eigenvalue equation. There exists quite a broad litterature on this subject ${ }^{i 2}$. Here we will rather follow the presentation of reference (11).

Let us first Wigner-transform the Hamiltonian matrix $h\left(r, r^{\prime}\right)$ defined in cquation (12), into $h_{w}$ namely

$$
h_{w}(R, h k)=\int d s e^{i k s} h\left(R+\frac{s}{z}, R-\frac{s}{z}\right)
$$

where

$$
R=\left(r+r^{\prime}\right) / 2
$$

Then, one can expand $h_{u}$ up to second order in $h k$, which corresponds to an expansion on the range of the non locality (rather than to a semiclassical expansion ):

$$
h_{w}(R, h k)=h_{w}(R, 0)+\frac{\hbar^{2}}{2} k^{2} F(R)
$$

where

$$
p=\hbar_{k}, F(R)=\left.\frac{\partial^{2} h_{w}}{\partial p^{2}}\right|_{(R, 0)}
$$

Upon inverse Wigner-transforming $h_{w}$

$$
h\left(r, r^{\prime}\right)=\frac{1}{2 \pi} \int d k e^{-i k\left(r-r^{\prime}\right)} h_{w}\left(\frac{r+r^{\prime}}{2}, \hbar k\right)(17)
$$

ore gets with the truncated $h_{w}$ of equation (15):

$$
h\left(r, r^{\prime}\right)=V\left(\frac{r+r^{\prime}}{2}\right) \delta\left(r-r^{\circ}\right)-\frac{\hbar^{2}}{2} F\left(\frac{r+r^{0}}{2}\right) \delta^{\prime \prime}\left(r \cdot r^{\prime}\right)
$$

where $V(r)$ is the diagonal matrix element $h(r, r)$.

Inserting the matrix $h\left(r, r^{\prime}\right)$ of equation (18) into equation (11) ( the eigenvalue equation for $\left.g_{\alpha}\right)$, one gets the Schrödinger equation:

$$
\begin{aligned}
& \left\{-\frac{\hbar^{2}}{2} \frac{\partial}{\partial r} F(r) \frac{\partial}{\partial r}\right. \\
& \left.+\left[V(r)-\frac{\hbar^{2}}{8} \frac{\partial^{2} F(r)}{\partial r^{2}}\right]-E_{\alpha}\right\} g_{\alpha}=0
\end{aligned}
$$

It clearly corresponds to a quantized version of the Bohr collective Hamiltonian upon considering $F(r)$ as an inverse mass and $V(r)$ as a potential. One notices the existence of a corrective tem to the potential. proportional to the second derivative of $B(r)$. The latter is usually roferred to as a Zero Point Encrg! ( ZPE) corrective tem. Now this Boln handilonian eigenvalue equation is 
defined in terms of $h(a) h_{u}$ ). To comnect this equation with what is prinarily. calculated ( $i$. e. the natrix $\mathcal{H}$ ) one generaliy relies on the Gaussian (Overlat) Appoximation ( $G(O A)$ of the GCM. We will wot treat that point here and merely refere to the previnusly anoted litteratime.

To smmuarize this Sub-Section, one should mention that with the preceding approach. one gets a quantized Bohr Hamiltonian with well defined ZPE (quantal corrections. On the other hand one should also adrl that a part of the approach relies on the validity of the GOA to the GCM, for which some elements of assessment are now avalable ${ }^{12}$. Moreover, the GCM is known ${ }^{13}$ ) to yield mass parameters that are not correct unless double GCM calculations are performed.

\section{I1.2 DERIVATION FRON THE ATDHFB METHOD}

Among the many derivations of a Bohr collective Hamiltonian from the Adiabatic Time-Dependent Hartree-Fock-Bogolyubor ( ATDHFB) method we will concentate on the approach initiated by Baranger and Vénéroni ${ }^{14,15)}$. Here also for the sake of clarity we will consider only one single collective variable even though it is easily extended to more. Similarly we will skip the pairing correlations and briefly present merely the ATDHF version (no pairing included) of this derivation. A generalisation in order to treat these correlations is possible and has indeed been achieved ${ }^{16)}$.

One starts from the TDHF equation of motion

$$
[h, \rho]=i \hbar \dot{p}
$$

$(20)$

Where the one-body Hamiltonian $h$ is understood as $h(\rho)$, i.e. defined in terms of the one-body reduced density matrix $\rho$. Now one makes an expansion in some velocities ( see references (14.15) for more details):

$$
\rho=\rho_{0}+\rho_{1}+\rho_{2}+\cdots
$$

(21).

Conversely one can make the same expansion for $h(\rho)$ and get:

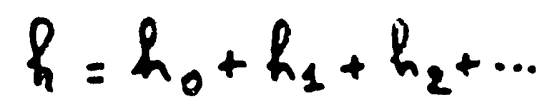

(22).

All quantities with an even (odd resp. ) subscript are even (ord resp. ) with respect to the tine-reversal operator. Separating the time-eren and timeorid parts of the equation of motion (20) one gets two equations. Tle first 1 the even one $)$ is nswally weferged to as the path equation. Its solution will be assumed to be well represented ly maling the following appoxinations:

i) all the tine-dependence of $p$ is contained in a single collective variable $q$. i. $e$

$$
e^{(t)} \rho[q(t)], \quad \dot{e}=\dot{q} \frac{\partial \rho}{\partial q}
$$


and therefore one has

$$
\rho_{0} \propto \dot{q}^{0}, \rho_{1} \propto \dot{q}^{1}, \rho_{2}-\dot{q}^{2} \quad \text { (24), }
$$

ii) the family $\rho_{0}(q)$ is defined from Constrained Hartree-Fock ( CHF ) calculations, namely

$$
\left[h_{0}-\lambda Q, \rho_{0}\right]=0, q=t\left(\rho_{0} Q\right) \text { (25). }
$$

Then it can be shown ${ }^{15)}$ that the time-odd equation takes the form of a doubly Constrained Hartree-Fock equation

$$
\left[h_{0}+h_{1}-\lambda Q-\dot{q} P_{1} \rho_{0}+l_{1}\right]=0
$$

where one adds to the constraint on $\langle Q\rangle$, a constraint on the expectation value of an operator $P$ which is univocally defined in terms of $\rho_{0}(q)$ ( and its derivative with respect to $q$ ) and which can be shown to be conjugate ( in a classical sense) of the operator $Q$ :

$$
\operatorname{ta}(\rho[Q, \rho])=i \hbar
$$

Having solved the double CHF problem, one na! compute the energy associated with the solution $p=\rho_{0}+\rho_{1}+\rho_{2}$ as an expansion again in $\dot{q}$

$$
E[\rho]=E\left[\rho_{0}\right]+\frac{1}{2} M(q) \dot{q}^{2}
$$

(28).

In the above equation $E\left[\rho_{0}\right]$ is the potential energy, as resulting from the simple CHF equation ( i.e. with a mere conitraint on $\langle Q\rangle$ ) and $M(q)$ is it mass parameter which in general depends on $q$ and is proportional to $\operatorname{tr}(\rho P / \dot{q})$

It is therefore clear that we have been able to yield a classical Bohr Hamiltonian. The derivation which we have slietched here, provides a generalization of the Thouless-Valatin formalism ${ }^{17}$ ) (or routhian formalism ) to collective modes other thar. pure rotations. As opposed to the GChl. one obtairs here good ma: : paranciers ${ }^{14}$ ) and furthermore fuli self-consistency corrections (beyond the Inglis cranking formula ${ }^{18}$ ) are appropriately incorporated.

On the other hand this classical Hamiltonian nust obviously be quantized and a choice has to be made on the ZPE comective terms. For those operations. as alrearly pointed out, one lacks a priori theoretical guidance within the mere ATDHFB framework.

III. A SAMPLE OF RESILTS 


\section{III.1 TRANSITIONAL NUCLEI}

The calculations reported in this Sub-Section have been performed within the ATDHFB framework described in Sub-Secion II.2. The Bohr Hamiltonian has been quantized according to the Pauli prescription with no ZPE corrections included. The CHF calculations have used the Skyme SIII effective nucleonnucleon interaction ${ }^{19)}$. Pairing corrclations have been taken care of by means of a BCS approach with constant pairing matrix elements. Whereas seif-consistent $P$ operators have been computed and used, one has merely here evaluated Inglis cranking mass parameters. Moreover the so-called Expectation Value Method has been substituted to the full HF plus BCS approach for the static calculations, making use of self-consistent semiclassical solutions (including terms up to the fourth order in $\hbar$ in an expansion a la Wigner- Kirkwood) associated with the considered two-body force. Some of the results discussed here, have already. been published elsewhere ${ }^{21}$.

Two different regions of transitional nuclei will be considered here $(A \sim 70$ and $A \sim 180$ ).

The low-lying experi-nental spectra of ${ }^{74} \mathrm{Ge}$ and ${ }^{70} \mathrm{Se}$ are qualitatively wellreproduced ( see reference (21), figure 2 ). A n interesting output of such calculations is obtained when plotting the probability density in the collective plane $(\beta, \gamma)$, namely plotting $\left|\phi_{\alpha}(\beta, \gamma)\right|^{2}$. In that respect, one should bear in mind that in order to have directly interpretable density plots, one generally considcrs cartesian integration measures for the density functions. To that effect the eigenfunctions must be multiplied by a factor proportional to $\left(\Im_{1} \Im_{2} \Im_{3}\right)^{1 / 2}$ which identically vanishes for $\gamma=n \pi / 3,(n=1, \ldots, 6)$. Therefore, a nucleus which should be considered as rather prolate (oblate resp. ) will have a maximum of the density near the $y=0$ axis $(\gamma=\pi / 3$ resp. ) but will of course never have such a maximum on this axis.

In the ${ }^{i 4}$ Ge nucleus, one has obtained ( see reference (21), figure 3 ) density distributions almost unaltered at low spin within the ground-state band (no centrifugal stretching or anti-stretching ) and a one-phonon state in the so-called $\beta$-band which exhibits the expected feature of a single node near the $\gamma=0$ axis. (In the absence of results on transition probabilities, band assignments have been made by mere energy considerations confirmed, if needed, by a comparison of the $\mathrm{K}$-component content.)

Systematical calculations in the platinum region are currently performed. The excellent result obtained for the ${ }^{180} \mathrm{Pt}$ has been aiready reported ( see reference (21), figure 2 ). We have extended such calculations to the ${ }^{190} \mathrm{Pt}$ and ${ }^{176} \mathrm{Pt}$ nuclei. Whercas for the former - as seen on figure 1 - the agrement with experimental energies is very satisfactory, it is found to be hardly the case for the ${ }^{170} \mathrm{Pt}$ where the first $2^{+}$level is significantly too low. missing thus the orcurence of a new transitional region below ${ }^{178} \mathrm{Pt}$ as suggested by the data.

The density probability contours of the ${ }^{180} \mathrm{Pt}$ exhibit a rery interesting plenomenon. Whereas the yrast band, starts with a mather oblate shape for 
$I=0,2$, this shape becomes prolate for $I \geq 4$. Conversely, the so-called $\beta$-band starts as a one-phonon state for $I=0$ and gradually corresponds to an oblate solution similar to what had been obtained for the ground state. This is thus a quantitative illustration of the shipe transition often advocated in this region.

\section{III.2 SUPERDEFORMATION AT LOM SPIN}

The calculations which will be briefly reported here, have been obtained by two of us ( I. D. and J. L. ) in collaboration with J.P. Delaroche and M. Girod. The results at spin zero have been already published ${ }^{22)}$. They correspond to the approach discussed in Sub-Section II.1. Static calculations have been performed within the HFB framework using the D1S Gogny effective force ${ }^{23)}$ which is not a corrected zero-range force as the Skyrme force and reproduces well at the same time HF-like and BCS-like matrix elements. To approximately take crire of the problem of the GCM masses, the mass parameters which have been included, are of the Inglis type ( making use however of simplified non self-consistent $P$ operators ).

The existence of superdeformed local minima in the potential energy surfaces in the region $A \sim 190$ has been predicted long ago both in Strutinsky -like calculations $^{24)}$ or in HF plus BCS calculations ${ }^{25)}$. ii. has been recently revisited theoretically ${ }^{26-28)}$ and the theoretical predictions have been confirmed afterwards experimentally ${ }^{29}$ ) ( see the contribution of E. Henry to this Conference fo: an update of this fast growing experimental domain ${ }^{30)}$ ).

The characteristic feature of potential cnergy curves (for axially symmetrical shapes ) in the region of nuclei with $A \sim 190$ is the existence of a pochet in the ascending part of the fission barrier which corresponds roughly to a mass quadrupole moment of about 45 barns. The relevant energy parameters are the e:acitation ( in a purely static sense here, with the inclusion of ZPE corrective tems though) energy $E^{*}$ and the inner barricr energy $E_{B}$ (measured from the ground state ). Upon including ZPE corrective terms these energies have been calculated to be ( see reference (22), figure 1 ) in ${ }^{190} \mathrm{Hg},{ }^{192} \mathrm{Hg}$ and ${ }^{194} \mathrm{Hg}$ :

$E^{*} \sim 1.5,3.0,5.0(\mathrm{MeV})$

$E_{13} \sim 5.5,7.0,9.5(\mathrm{MeV})$.

Inserting full potential energy surfaces as well as the calculated mass parameters and moments of inertia, one gets eigensolutions of the corresponding Bohr Hamiltonians which present the following features for $\mathrm{c}$ g. the $0^{+}$states: i) ground states ypical of transitional ( slightly) oblate nuclei, ii) at values of excitation energies of $4.4,5.4,6.3 \mathrm{MeV}^{\prime}$ for the ${ }^{190} \mathrm{Hg},{ }^{192} \mathrm{Hg}$ and ${ }^{194} \mathrm{Hg}$ nuclei, one gets states that are mostly located in the iscmeric well (see refercnce (22): figure 4 ). To be specific, if one considers the part of the probability in the collective space corresponding to $3 \geq 0.4$, one gets $91 \%, 98 \%$ and $36 \%$ for each of the abore listed nuclei in this particular state. These conclusions have recently been extended to finite spins. Indeed rotational bands built on the previous $0^{+}$ states are found. In ${ }^{190} \mathrm{Hg}$, the corresponding superdeformed band cresses the 
yrast ( normal) band around $I=10,12$. This spin value seems to be too low in view of recent experimental data $^{30)}$ and can reflect the fact that the static excitation energy $\left(E^{*}\right)$ values in these calculations are found significantly lower than in other dynamical calculations ${ }^{12,31)}$.

\section{CONCLUSION}

Two different derivations in a microscopic fashion of collective Bohr Hamiltonians have been recalled. None of them is without problems. It is convenient at this point to answer the following question: aiter all why not go on and perform double GCM calculations which would be purely quantal and free from some mass problems? It is unfortunate that at this time, one is at best able to make two dimensional GCM calculations ${ }^{12)}$. On the other hand, to treat correctly the quadrupole motion which carries most of the low-energy collective motion, one needs, as it is well known and recalled here, to consider five degrees of freedom. This is the reason why we must go through the above mentionned difficulties.

It is however clear that in the present status such calculations are able to yield a very good qualitative reproduction of data. How quantitative the qualitative agreement is indeed, time will tell. It is particularly appropriate in this context to emphazise the crucial role played by pairing correlations for which such calculations constitute a very demanding test. The obtained reproduction of experimental spectra for rough that it may be deemed, is nevertheless very significant in view of the absence of any ad-hoc parameter fitting except for the torce parameters chosen many years ago in a different context, i. e. to reproduce essentially saturation properties ( let us also recall that apart from two pairing parameters, the Skyme SIII force has only six parameters for the whole chart of nuclides ). Finally, the obvious point where some effort should be devoted is undoubtedly an improvement of the mass parameters in use which are presently somewhat too crude. 


\section{REFERENCES}

+ This work was supported in part by the U.S. Department of Eneray by Lawrence Livermore National Laboratory under contract no. W-7405-ENG-48.

1. A. Bohr, Mat.Fy's. Medd. Dan Vid. Selsk. 20 (1952) \# 14.

2. K. Kumar and M. Baranger, Nucl. Phys. A92 (1967) 608.

3. K. Kumar, Nucl. Phys. CL (1974) 189.

4. J. Libert and P. Quentin, Z. Phy's. A306 (1982) 315.

5. D.L. Hill and J.A. Wheeler, Phys. Rev. 89 (1953) 1102.

6. see e. g. P. Ring and P. Schuck, The Nuclear Many-Body Problem, Springer Verlag, Berlin (1980).

7. D. M. Brink and A. Weiguny, Nucl. Phys. A120. (1968) 59.

8. B. Giraud and B. Grammaticos, Nucl. Phys. A233 (1974) 373.

9. P.G. Reinhard, Nucl. Phys. A252 (1975) 120.

10. M. Girod and B. Grammaticos, Nucl. Phys. 1233 (1979) 40.

11. J.F. Berger, Thesis, Orsay (1985), unpublished.

12. P. Bonche, J. Dobaczewski, H. Flocard, P.-H. Heenen and J. Meyer, Nucl. Phys. A510 (1990) 466 .

13. R. E. Peierls and D. J. Thouless, Nucl. Phỵs. 38 (1962) 154.

14. M. Baranger and M. Vénéroni, Ann. Phys. (New York) 114 (1978) 123.

15. M.J. Giannoni and P. Quentin, Phys. Rev. C.21 (1980) 2060.

1C. L. Bennour, J. Libert and P. Quentin, to be published.

17. D.J. Thouless and J.G.Valatin, Nucl. Phys. 31 (1962) 211.

18. D.R. Inglis, Phys. Rev. 96 (1954) 1059; 103 (1956) 1796.

19. M. Beiner, H. Flocard, Nguyen Van Giai and P. Quentin, Nucl. Phys. A23S (1975) 29.

20 I. Deloncle, K. Bencheikh, L. Bennour, M. Brack, J. Libert, J Meyer and P. Quentin, to be published.

21 I. Deloncle, J. Libert, L. Bennour and P. Quentin, Phys. Lett. B233 (19S9) 16.

22. J.P. Delaroche, M. Girod, J. Libert and I. Deloncle, Phys. Lett. B232 (19S3) 145.

23 J. Dechargś and D. Gogny, Phỵs. Rev. C21 (1980) 1568; J.F.Berger, M. Girod and D. Gogny, Nucl. Phys. A428 (1982) 23c.

24. R.R. Pashkevitch, preprint JINR (Dubna) Pł-4383 (1969), unpublished.

25. M. Cailliau, J. Letessier, H. Flocard and P. Onentin, Plyys. Lett. B46(1973) 11.

26. P. Bonche, S.J. Krieger, P. Quentin, M.S. Meiss, J. Meyer. M. Meyer. N. Redon. H. Flocard, and P.-H. Heenen, Nucl. Plyss. 4500) (1989) 308.

27. M. Girod, J.P. Delaroche, D. Gogny and J.F. Berger, Phys. Rev. Lett. 62 (1989) 2452.

28. R.R. Chasman, Phys. Lett. B219 (19S9) 227. 
29. E.F. Moore, R.V.F. Janssens, R.: Chasman, I. Ahmad, T.L. Khoo, F.L.H. Wolfs, D. Ye, K.B. Feard, U. Garg, M.W. Drigert, Ph. Benet, Z.W. Grabowski and J.A. Cizewski. Phys. Rev. Lett. 63 (1989) ¿00.

30. E.A. Henry, invited taik to t. s Symposium.

31. P. Bonche, S.J. Irieger, M.S. Neiss, J. Dobaczewsli, H. Flocard, P.-H. Heen $\circ$ and $i$. Meyer, invited talk to this Symposium. 


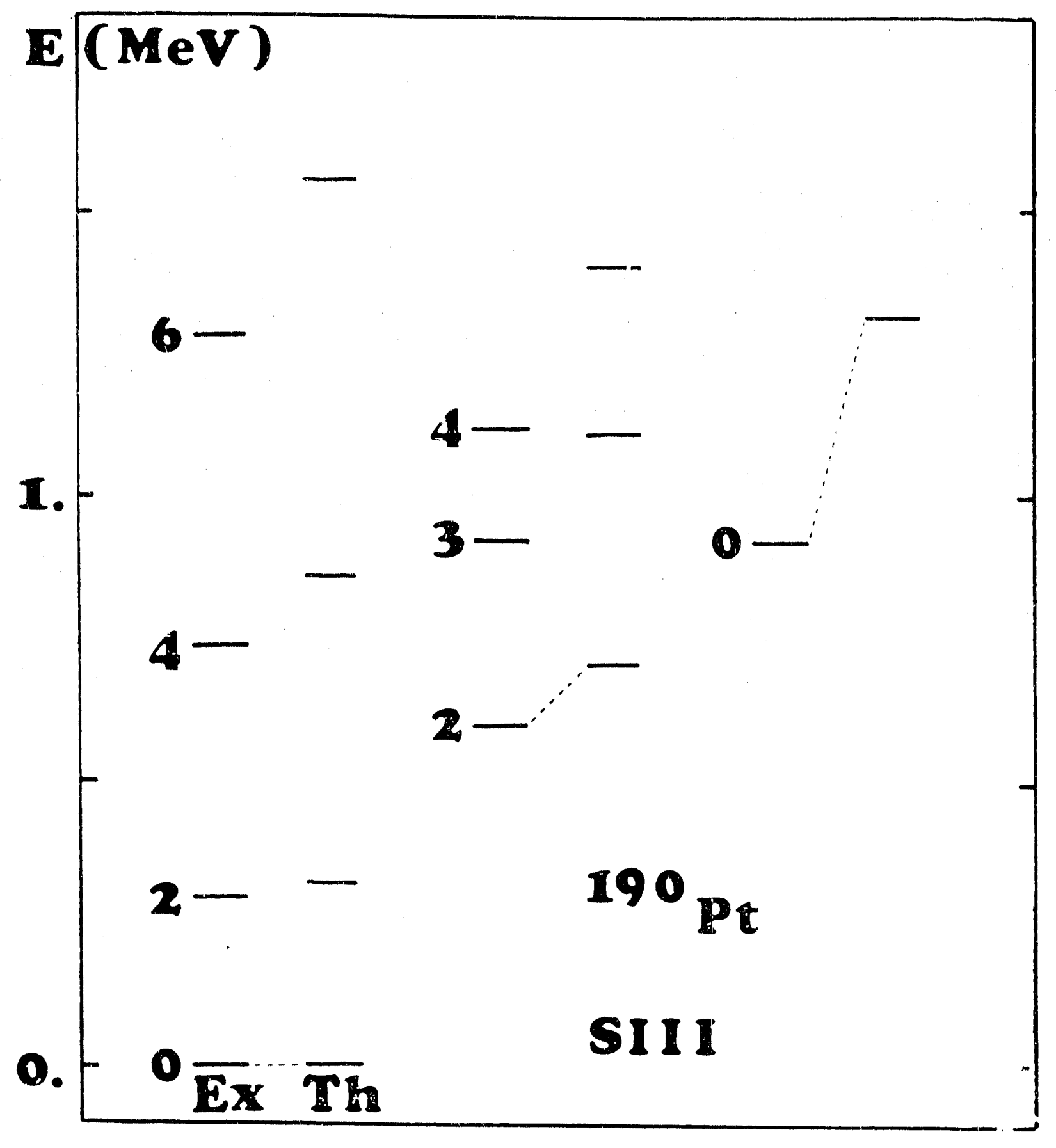

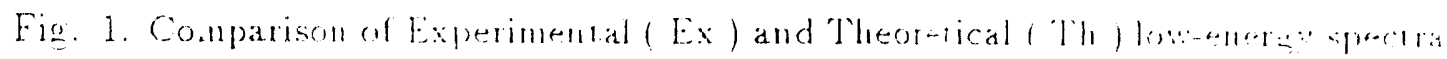

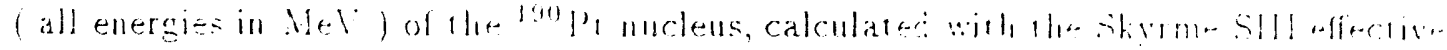
intaraclion. 

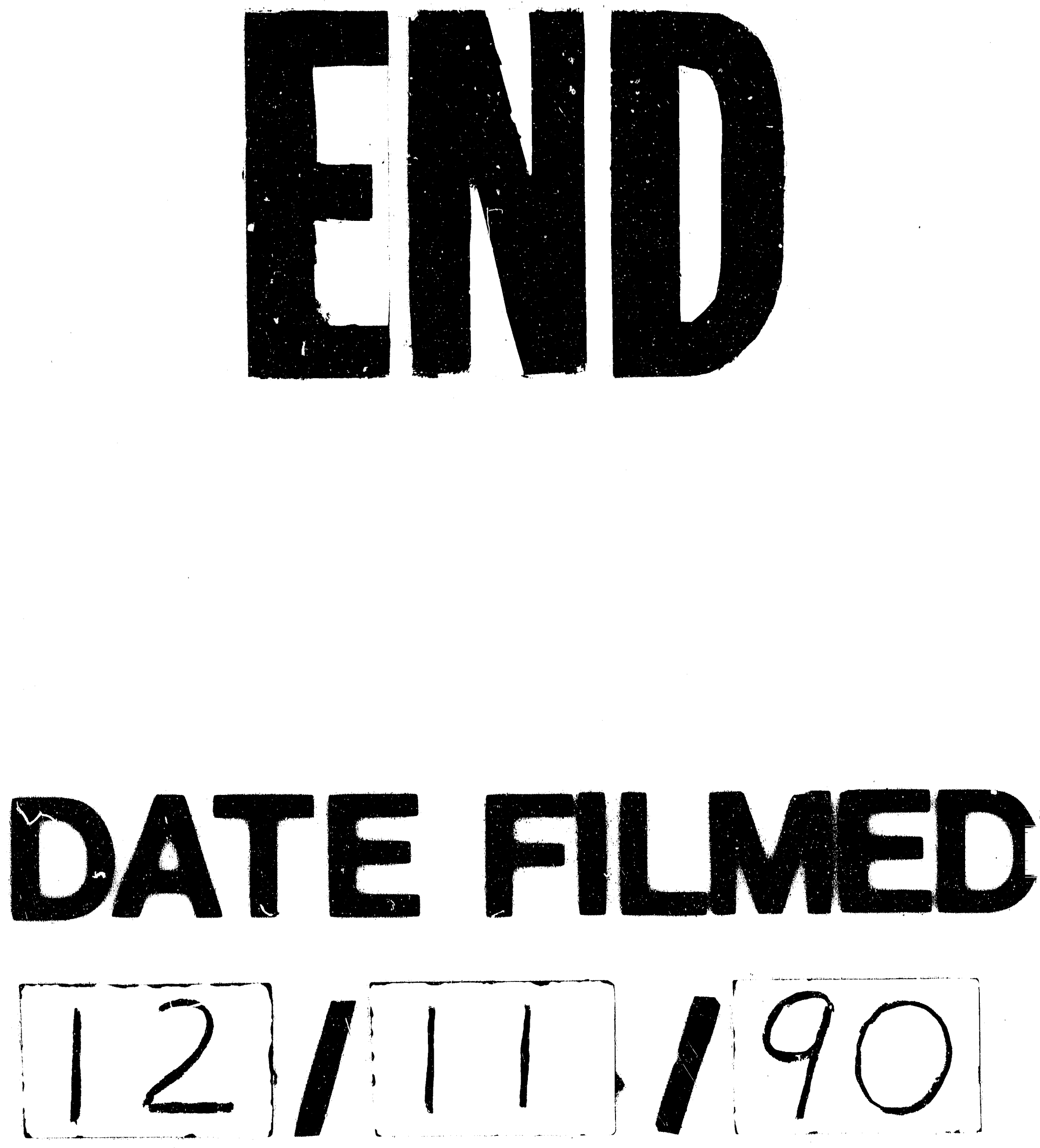
